\title{
Severe Back Pain in a Young Patient with Pyoderma Gangrenosum and Crohn's Disease Controlled with Anti-tumor Necrosis Factor Therapy: Sterile Osteomyelitis
}

\author{
Sarah Felton · Firas Al-Niaimi · Calum Lyon
}

To view enhanced content go to www.dermtherapy-open.com Received: December 16, 2013 / Published online: February 5, 2014

(c) The Author(s) 2014. This article is published with open access at Springerlink.com

\section{ABSTRACT}

Introduction: Inflammatory bowel disease has been associated with a number of cutaneous and systemic neutrophilic disorders, including pyoderma gangrenosum. In 1972, the term chronic multi-focal recurrent osteomyelitis was given to a sterile neutrophilic condition which has been associated with inflammatory bowel disease.

Case Report: We report a case of a 23-year-old man with long-standing severe Crohn's disease which necessitated subtotal colectomy. He subsequently developed progressive, intermittent back pain that were limiting his functional movement. Numerous investigations to identify what initially was thought to be an infectious process failed to lead to the diagnosis.

Electronic supplementary material The online version of this article (doi:10.1007/s13555-014-0044-3) contains supplementary material, which is available to authorized users.

S. Felton · F. Al-Niaimi $(\square)$

Department of Dermatology, Salford Royal

Foundation Trust, Manchester, UK

e-mail: firas55@hotmail.com

C. Lyon

Department of Dermatology,

York Hospital, York, UK
Biopsy of the spine identified a sterile neutrophilic infiltrate and the diagnosis of chronic recurrent multi-focal osteomyelitis was made which was successfully treated with immunosuppressive drugs.

Conclusion: Inflammatory bowel disease can present with cutaneous and systemic neutrophilic disorders and this association is becoming increasingly recognized by gastroenterologists and dermatologists. Chronic recurrent multi-focal osteomyelitis is a sterile neutrophilic disorder which can present with bone pain and responds to immunosuppressive therapy.

Keywords: Anti-tumor necrosis factor therapy; Azathioprine; Chronic recurrent multi-focal osteomyelitis; Crohn's disease; Clobetasol; Dermatology; Metronidazole; Prednisolone; Pyoderma gangrenosum; Tacrolimus

\section{INTRODUCTION}

Immuno-suppressed patients are prone to a number of bony disorders including infective osteomyelitis and spinal lymphoma [1]. The authors describe their experience of the 
investigation and management of back pain in a patient with inflammatory bowel disease (IBD) and pyoderma gangrenosum (PG), with the subsequent diagnosis of chronic recurrent multi-focal osteomyelitis (CRMO). Noninfectious causes of bone pain have been reported in certain neutrophilic disorders and dermatologists should be aware of this rare, but otherwise debilitating syndrome.

\section{CASE REPORT}

A 23-year-old male patient with long-standing Crohn's disease, which had necessitated subtotal colectomy, was under regular dermatological review for the management of hidradenitis suppurativa, perianal Crohn's disease and recalcitrant PG affecting the inguinal and scrotal regions. He was treated with a combination of topical tacrolimus ointment at the strength of $0.3 \%$ mixed with clobetasol ointment, oral metronidazole, oral prednisolone and azathioprine in an attempt to control bowel and skin symptoms. These resulted in limited long-term benefit and treatment with biological therapy infliximab was considered. At the same time, the patient also described the progressive development of intermittent mid-back pain that did not settle with a combination of analgesics (paracetamol, tramadol, diazepam and ibuprofen).

Spinal X-rays had not shown any abnormality but he was admitted acutely for severe unrelenting back pain, and a significantly elevated C-reactive protein (CRP) level of $350 \mathrm{mg} / \mathrm{L}$, which coincided with acute worsening of the cutaneous PG. A bone scan highlighted abnormally intense activity within some thoracic (T) vertebrae; bone windows on computerized tomography (CT) revealed soft tissue thickening on T7 and T9 vertebral bodies, with a small amount of adjacent bony lucency related to the $\mathrm{T} 9$ area; magnetic resonance imaging (MRI) demonstrated multi-level $\mathrm{T}$ vertebral body abnormalities, but no soft tissue masses, vertebral fractures or evidence of discitis. The aforementioned features were suggestive of spinal lymphoma or chronic osteomyelitis. Concern was raised about the use of anti-tumor necrosis factor (TNF) treatments for his PG and Crohn's disease because of the potential for worsening what was a possible infective osteomyelitis. A spinal biopsy was, therefore, performed and histology described thickened trabecular bone with woven bone formation on polarized light, and some osteoblastic activity. The bone marrow in some areas was fibrotic with small thin-walled vessels, but there was no granulomatous inflammation or evidence of neoplasia. Appearances were in keeping with chronic, non-specific osteomyelitis, but cultures from biopsy material were negative for bacteria, mycobacteria and fungi. The clinical presentation and absence of microbiological agents led to the diagnosis of CRMO.

Once infection had been excluded, the patient was treated with prednisolone and azathioprine, while awaiting anti-TNF therapy. Infliximab substantially improved skin and bowel symptoms. In addition, two subsequent spinal MRI scans at 9 and 18 months have shown resolution of the signal changes within the vertebral bodies, coinciding with marked resolution of the clinical symptoms of back pain.

All procedures followed were in accordance with the ethical standards of the responsible committee on human experimentation (institutional and national) and with the Helsinki Declaration of 1975, as revised in 2000 and 2008. Informed consent was obtained from all patients for being included in the study. 


\section{DISCUSSION}

The term CRMO was first described in 1972 as an unusual form of multi-focal bone lesions encompassing subacute and chronic symmetrical osteomyelitis $[1,2]$. It is characterized by self-limiting but relapsing episodes of non-infectious osteomyelitis. Although of unknown etiology, CRMO has recognized association with a number of dermatoses, particularly the neutrophilic dermatoses of PG, Crohn's and Sweet's syndrome $[3,4]$. Its association with IBD was first reported in 1998 [5], and the diagnosis may precede the development of bowel symptoms by around 5 years [6]. More recently, it has been suggested that CRMO may represent a pediatric form of SAPHO (synovitis, acne, pustulosis, hyperostosis, osteitis), and should be considered as an auto-inflammatory syndrome [7].

Patients are usually children who present with recurrent episodes of bone pain, general malaise and raised inflammatory markers, which may be initially misdiagnosed as infective osteomyelitis or neoplasia [8]. The main sites of reported involvement are the tibia, pelvis, femur and clavicle [8]. Nonsteroidal anti-inflammatory drugs are often used as first-line treatment with good response. Second-line options include bisphosphonates, systemic immunosuppressive drugs and rarely, such as in our case, TNF antagonists $[6,8,9]$. While oral steroids are effective in the majority of cases they are generally not used due to the side effects of long-term treatment. Follow-up studies have shown that the disease can remain active for around 10 years, highlighting its chronic and recurrent nature [10].

The author wishes to highlight the increasingly recognized phenomenon of
CRMO and in particular document the previously unreported correlation between episodes of severe back pain and flares of PG. It is inevitably important to consider CRMO in the differential diagnosis of back pain as treatment options involve immunosuppression rather than the high-dose prolonged antibiotic therapy required for infective osteomyelitis. In this patient, anti-TNF therapy that was originally instigated for control of bowel symptoms also ameliorated PG and his back pain due to CRMO.

\section{CONCLUSION}

In conclusion, we report a case of a young man with the background of PG and IBD who presented with severe back pain as part of a rare condition of CRMO. Accurate identification and diagnosis of this condition enables timely treatment with minimal sequelae.

\section{ACKNOWLEDGMENTS}

All named authors meet the ICMJE criteria for authorship for this manuscript, take responsibility for the integrity of the work as a whole, and have given final approval for the version to be published. No funding or sponsorship was received for this study or publication of this article.

Conflict of interest. F. Al-Niaimi, S. Felton and C. Lyon declare no conflicts of interest.

Compliance with ethics. All procedures followed were in accordance with the ethical standards of the responsible committee on human experimentation (institutional and national) and with the Helsinki Declaration of 1975, as revised in 2000 and 2008. Informed 
consent was obtained from all patients for being included in the study.

Open Access. This article is distributed under the terms of the Creative Commons Attribution Noncommercial License which permits any noncommercial use, distribution, and reproduction in any medium, provided the original author(s) and the source are credited.

\section{REFERENCES}

1. Girschick HJ, et al. Chronic recurrent multifocal osteomyelitis: what is it and how should it be treated? Nat Clin Pract Rheumatol. 2007;3(12):733-8.

2. Giedion A, Holthusen W, Masel LF, Vischer D. Subacute and chronic "symmetrical" osteomyelitis. Ann Radiol. 1972;15:329-42.

3. Matsumura $\mathrm{Y}$, et al. Pyoderma gangrenosum in a patient with myelodysplastic syndrome followed by possible extracutaneous manifestations in the gallbladder, liver, bone and lung. J Dermatol. 2011;38(11):1102-5.

4. Morbach $\mathrm{H}$, et al. Association of chronic nonbacterial osteomyelitis with Crohn's disease but not with CARD15 gene variants. Rheumatol Int. 2010;30(5):617-21.

5. Bognar M, Blake W, Agudelo C. Chronic recurrent multifocal osteomyelitis associated with Crohn's disease. Am J Med Sci. 1998;15:133-5.

6. Deutschmann A, et al. Successful treatment of chronic recurrent multifocal osteomyelitis with tumor necrosis factor-alpha blockage. Pediatrics. 2005;116(5):1231-3.

7. Tlougan BE, Podjasek JO, O'Haver J, et al. Chronic recurrent multifocal osteomyelitis (CRMO) and synovitis, acne, pustulosis, hyperostosis, and osteitis (SAPHO) syndrome with associated neutrophilic dermatoses: a report of seven cases and review of the literature. Pediatr Dermatol. 2009;26:497-505.

8. Wipff J, Adambaum C, Kahan A, Job-Deslandre C. Chronic recurrent multifocal osteomyelitis. Joint Bone Spine. 2011;78(6):555-60.

9. Bret J, et al. Aseptic osteomyelitis responding to TNF alpha antagonist therapy in a patient with Crohn's disease. Joint Bone Spine. 2008;75(4):489-91.

10. Huber AM, Lam PY, Duffy CM, et al. Chronic recurrent multifocal osteomyelitis: clinical outcomes after more than five years of follow-up. J Pediatr. 2002;141:198-203. 